\title{
PECULIAR KINEMATICS IN THE CORE OF NGC 474
}

\author{
G. K. T. HAU ${ }^{1}$, M. BALCELLS ${ }^{2} \&$ D. CARTER ${ }^{3}$
}

${ }^{1}$ Inst. of Astron. $\mathcal{E}^{3}{ }^{3}$ Royal Greenwich Obs., Cambridge, UK

${ }^{2}$ Kapteyn Laboratorium, Groningen, The Netherlands
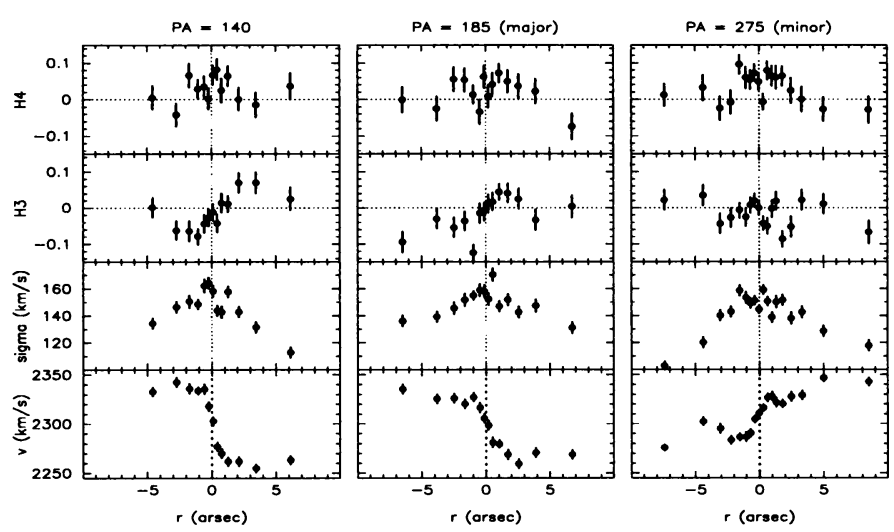

We present absorption line profile analysis of NGC 474, an elliptical with prominent, irregular shells. Profiles are parameterized with GaussHermite polynomials (van der Marel \& Franx ApJ 407 1993; Rix \& White MNRAS 254 1992). The fastest rotation $\left(\sim 50 \mathrm{~km} \mathrm{~s}^{-1}\right)$ and steepest central velocity gradient along the intermediate photometric axis rules out the possibility that NGC 474 is a face-on S0 (Schombert \& Wallin AJ 94 $1987)$ and suggests that it is triaxial. The asymmetry of the LOSVDs ( $h 3$ up to 0.08 ) indicates the presence of a subsystem with rapid, ordered rotation. The minor axis velocity curve shows a kinematic feature at 3-4" east of the nucleus, with no associated $h 3$ or $h 4$ features. Non-parametric LOSVD analysis (unresolved Gaussian decomposition, Kuijken \& Merrifield MNRAS 264 1993) reveals a double-peaked profile at that location. In all position angles line-profiles are distinctly pointy for radii up to $2 "$, and are consistent with zero further out. We have found similar central positive $h 4$ terms in the shell galaxy NGC 2865 (Hau et al. MNRAS in prep). Cores with pointy LOSVDs are uncommon in ellipticals (Bender, Saglia \& Gerhard MNRAS 269 1994). Positive $h 4$ terms might contain important clues on the shell-formation mechanism in ellipticals. 